\title{
TPM3-NTRK1 fusion in a pleomorphic xanthoastrocytoma presenting with haemorrhage in a child
}

\author{
Katherine Clark Pehlivan, ${ }^{1}$ Denise M Malicki, ${ }^{2}$ Michael L Levy, ${ }^{3}$ John Ross Crawford ${ }^{4}$
}

${ }^{1}$ Neurosciences, University of California San Diego, San Diego, California, USA

2Pathology, Rady Children's Hospital University of California San Diego, San Diego,

California, USA

${ }^{3}$ Neurosurgery, University of California San Diego, San Diego, California, USA

${ }^{4}$ Neurosciences and Pediatrics, University of California San Diego, San Diego, California, USA

\section{Correspondence to} Dr John Ross Crawford; jrcrawford@ucsd.edu

Accepted 9 February 2020

\section{DESCRIPTION}

A previously healthy 6-year-old boy presented with acute onset of headache, vomiting and right sided weakness. CT imaging was performed which revealed an intracerebral left-sided $7 \mathrm{~cm}$ haemorrhagic mass associated with midline shift (figure 1). The patient subsequently developed signs of herniation syndrome and an emergent evacuation of the hematoma and a gross total tumour resection was performed. The tumour consisted of a moderately cellular proliferation of spindle cells with pleomorphic nuclei, which stained positive for glial fibrillary acidic protein (GFAP) and low Ki67, consistent with a diagnosis of pleomorphic xanthoastrocytoma (PXA), WHO Grade II (figure 2). A DNA-based next generation sequencing panel consisting of 397 cancer-related genes (table 1) performed on paraffin-embedded formalin fixed tumour demonstrated a TPM3 NTRK1 fusion between TPM3 exon 7 and NTRK1 exon 10 and loss of CDKN2A and CDKN2B.

PXA represents less than $1 \%$ of paediatric brain tumours. Upfront treatment generally involves surgical resection followed by observation. However PXAs may recur in about $40 \%$ of patients within 10 years. ${ }^{1}$ Molecularly, almost two-thirds of cases harbour a BRAF (v-raf murine sarcoma viral oncogene homolog B1) V600E mutation and malignant variants have been reported. ${ }^{2}$ To our knowledge, our case is the first reported TPM3NTRK1 fusion in a PXA. NTRK fusions are quite rare in paediatric low grade glioma (LGG), typically identified in $<1 \%$ of LGGs. Out of 461 samples of LGG sequenced through the cancer genome atlas, NTRK fusions were identified in only 2 and both of these involved NTRK 2 fusions. ${ }^{3}$ Interestingly this combination of TPM3-NTRK1

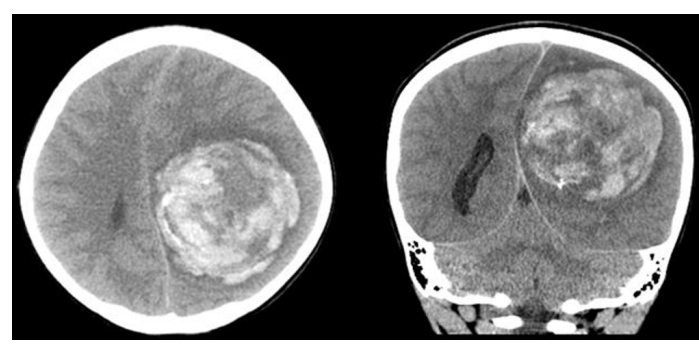

Figure 1 Axial and coronal non-contrast CT images reveal a haemorrhagic parenchymal mass in the posterior frontoparietal region associated with vasogenic oedema and midline shift.

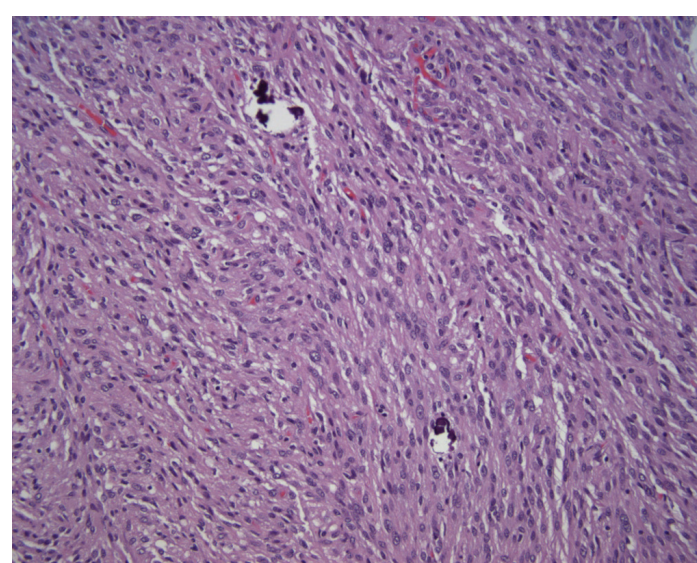

Figure 2 H\&E stained section of tumour at 200x magnification demonstrates cellular proliferation of spindle cells with pleomorphic nuclei consistent with a diagnosis of pleomorphic xanthoastrocytoma.

fusion and loss of CDKN2A and CDKN2B has been reported in one prior paediatric case, which was classified as an LGG, not otherwise specified. ${ }^{4}$

NTRK fusions represent a pharmacologically targetable genomic alteration. NTRK inhibitors are currently in clinical trials for patients with primary central nervous system tumours with promising results. ${ }^{5}$ Early results from a trial of larotrectinib has shown an overall response rate of $36 \%$ in 14 evaluable patients and tumour control rate of $71 \%$ of patients with stable disease or response. ${ }^{5}$ In the case of our patient who remains in remission, the use of an NTRK inhibitor would be considered a potential alternative to chemotherapy or radiation at time of recurrence. In summary, our case highlights a rare variant of PXA presenting with acute haemorrhage defined

\section{Learning points}

- Pleomorphic xanthoastrocytoma (PXA) may present with acute intracranial haemorrhage.

- NTRK fusions can occur in PXAs in paediatric patients.

- Although BRAF V600E mutations are the most common molecular aberration in PXAs, molecular genetic testing on these tumours may identify other pharmacologically targetable mutations 
Images in...

Table 1 Next generation cancer gene panel

\begin{tabular}{|c|c|c|c|c|c|c|c|c|c|c|c|}
\hline \multicolumn{12}{|c|}{ Genes for which entire coding panel is interrogated } \\
\hline$A B L 1$ & $A B L 2$ & ACVR1B & AKAP9 & AKT1 & AKT2 & AKT3 & $A L K$ & AMER1 & $A P C$ & $A R$ & ARAF \\
\hline ARFRP1 & ARID1A & $A R I D 1 B$ & ARID2 & ASPSCR1 & $A S X L 1$ & ATF6 & ATM & ATP1A1 & $A T P 2 B 3$ & ATR & $\operatorname{ATRX}$ \\
\hline AURKA & $A U R K B$ & AXIN1 & $A X L$ & BAP1 & BARD1 & $B C L 11 A$ & $B C L 11 B$ & $B C L 2$ & $B C L 2 L 1$ & $B C L 2 L 2$ & $B C L 6$ \\
\hline$B C O R$ & BCORL1 & $B L M$ & BMPR1A & $B R A F$ & $B R C A 1$ & $B R C A 2$ & BRD4 & BRIP1 & BTG1 & BTK & C11orf30 \\
\hline CACNA1D & CAMTA1 & CARD11 & CASP8 & $C B F B$ & $C B L$ & $C B L B$ & CCND1 & CCND2 & CCND3 & CCNE1 & CD274 \\
\hline$C D 79 A$ & $C D 79 B$ & $C D C 73$ & $\mathrm{CDH} 1$ & $\mathrm{CDH} 11$ & CDK12 & CDK4 & CDK6 & CDK8 & CDKN1A & CDKN1B & CDKN2A \\
\hline CDKN2B & CDKN2C & CEBPA & CHD2 & CHD4 & CHEK1 & CHEK2 & $C I C$ & CLTCL1 & COL1A1 & CREBBP & CRKL \\
\hline CRLF2 & CSF1R & CSF3R & CTCF & CTNNA1 & CTNNB1 & CUL3 & $C Y L D$ & $D A X X$ & DDIT3 & $D D R 2$ & DICER1 \\
\hline DNM2 & DNMT3A & DOT1L & EBF1 & $E G F R$ & EIF1AX & EP300 & EPHA3 & EPHA5 & EPHA7 & EPHB1 & ERBB2 \\
\hline ERBB3 & ERBB4 & $E R C C 3$ & ERCC4 & $E R C C 5$ & $E R G$ & ERRFI1 & ESR1 & ETV1 & ETV4 & ETV5 & ETV6 \\
\hline EWSR1 & EXT1 & $E Z H 2$ & FAM46C & FANCA & FANCC & FANCD2 & FANCE & FANCF & FANCG & FANCL & FAS \\
\hline FAT1 & FBX011 & FBXW7 & FGF10 & FGF14 & FGF19 & FGF23 & FGF3 & FGF4 & FGF6 & FGFR1 & FGFR2 \\
\hline FGFR3 & FGFR4 & $F H$ & FLCN & FLT1 & FLT3 & FLT4 & FOXA1 & FOXL2 & FOX01 & FOXP1 & FRS2 \\
\hline FUBP1 & FUS & GABRA6 & GATA1 & GATA2 & GATA3 & GATA4 & GATA6 & GID4 & GLI1 & GMPS & GNA11 \\
\hline GNA13 & GNAQ & GNAS & GPR124 & GRIN2A & GRM3 & GSK3B & H3F3A & $H G F$ & HIP1 & HNF1A & HRAS \\
\hline HSD3B1 & HSP90AA1 & $I D H 1$ & IDH2 & IGF1R & IGF2 & $I K B K E$ & $I K Z F 1$ & $I L 7 R$ & INHBA & INPP4B & IRF2 \\
\hline IRF4 & IRS2 & ITK & $J A K 1$ & $J A K 2$ & $J A K 3$ & JUN & KАТбA & KAT6B & KDM5A & KDM5C & KDM6A \\
\hline$K D R$ & KEAP1 & KEL & KIF5B & KIT & $K L H L 6$ & KMT2A & $K M T 2 D$ & KRAS & $\angle C P 1$ & LIFR & LMO1 \\
\hline$L R I G 3$ & $\angle R P 1 B$ & $L Y N$ & LZTR1 & MAGI2 & MAML2 & MAP2K1 & MAP2K2 & MAP2K4 & МАРЗК1 & MCL1 & MDM2 \\
\hline MDM4 & MED12 & MEF2B & MEN1 & MET & MITF & MLH1 & MLLT3 & MLLT4 & MN1 & $M P L$ & MRE11A \\
\hline MSH2 & MSH6 & MTOR & MUTYH & $M Y B$ & MYC & $M Y C L$ & MYCN & MYD88 & MYH11 & МYH9 & NCOA1 \\
\hline NCOA2 & NF1 & $N F 2$ & NFE2L2 & NFKBIA & NIN & $N K X 2-1$ & NOTCH1 & NOTCH2 & NOTCH3 & NPM1 & NR4A3 \\
\hline NRAS & NSD1 & NTRK1 & NTRK2 & NTRK3 & NUMA1 & NUP214 & NUP93 & NUP98 & PAK3 & PALB2 & PARK2 \\
\hline PAX3 & PAX5 & PAX7 & PBRM1 & PCM1 & PDCD1LG2 & PDGFRA & PDGFRB & PDK1 & PIK3C2B & PIK3CA & PIK3CB \\
\hline PIK3CG & PIK3R1 & PIK3R2 & PLCG2 & PMS1 & PMS2 & POLD1 & POLE & PPARG & PPP2R1A & PRDM1 & PRDM16 \\
\hline PREX2 & PRKAR1A & PRKCI & PRKDC & PRSS8 & PTCH1 & PTEN & PTPN11 & PTPRC & QKI & $R A C 1$ & $R A D 21$ \\
\hline RAD50 & RAD51 & RAF1 & RALGDS & RANBP17 & RANBP2 & RARA & $R B 1$ & RBM10 & RET & RICTOR & RNF43 \\
\hline ROS1 & RPTOR & RUNX1 & RUNX1T1 & SDHA & $S D H B$ & SDHC & $S D H D$ & SETBP1 & SETD2 & $S F 3 B 1$ & SLC34A2 \\
\hline SLIT2 & SMAD2 & SMAD3 & SMAD4 & SMARCA4 & SMARCB1 & SMO & SNCAIP & SOCS1 & sox10 & SOX2 & SOX9 \\
\hline SPEN & $S P O P$ & SPTA1 & $S R C$ & SRGAP3 & SS18 & STAG2 & STAT3 & STAT4 & STAT5B & STK11 & SUFU \\
\hline SYK & TAF1 & $T B X 3$ & TCF7L2 & TERC & TERT & TET2 & TGFBR2 & THRAP3 & TMPRSS2 & TNFAIP3 & TNFRSF14 \\
\hline TOP1 & TOP2A & TP53 & $T P R$ & TRIM24 & TRIM33 & TRIP11 & TRRAP & TSC1 & $T S C 2$ & TSHR & U2AF1 \\
\hline VEGFA & VHL & WHSC1 & WISP3 & WRN & WT1 & XP01 & ZBTB2 & ZMYM2 & ZNF217 & ZNF384 & ZNF521 \\
\hline \multicolumn{12}{|c|}{ Subset of genes (28) for which potential rearrangements are evaluated } \\
\hline$A L K$ & ASPSCR1 & $B R A F$ & BRD4 & DDIT3 & $E G F R$ & ETV1 & ETV4 & ETV5 & ETV6 & EWSR1 & FGFR1 \\
\hline FGFR2 & FGFR3 & FOX01 & FUS & MYB & NOTCH2 & NR4A3 & NTRK1 & NTRK2 & PDGFRA & PPARG & RAF1 \\
\hline RET & ROS1 & SS18 & TMPRSS2 & & & & & & & & \\
\hline
\end{tabular}

BRAF, v-raf murine sarcoma viral oncogene homolog B1.

by a novel TPM3-NTRK1 fusion that may be amenable to targeted therapy.

Contributors $\mathrm{KCP}, \mathrm{DMM}, \mathrm{MLL}$ and JRC were responsible for the design and writing of the manuscript and approval of its content.

Funding The authors have not declared a specific grant for this research from any funding agency in the public, commercial or not-for-profit sectors.

Competing interests None declared.

Patient consent for publication Parental/guardian consent obtained.

Provenance and peer review Not commissioned; externally peer reviewed.

\section{REFERENCES}

1 Rao AAN, Laack NN, Giannini C, et al. Pleomorphic xanthoastrocytoma in children and adolescents. Pediatr Blood Cancer 2010;55:290-4.

2 Ida CM, Rodriguez FJ, Burger PC, et al. Pleomorphic xanthoastrocytoma: natural history and long-term follow-up. Brain Pathol 2015;25:575-86.

3 Stransky N, Cerami E, Schalm S, et al. The landscape of kinase fusions in cancer. Nat Commun 2014;5:4846.

4 Johnson A, Severson E, Gay L, et al. Comprehensive genomic profiling of 282 pediatric low- and high-grade gliomas reveals genomic drivers, tumor mutational burden, and hypermutation signatures. Oncologist 2017;22:1478-90.

5 Ziegler DS, Doz F, Geoerger B, et al. Activity of larotrectinib in TRK fusion cancer patients with primary central nervous system tumours. Annals of Oncology 2019:30:ix124. 
Copyright 2020 BMJ Publishing Group. All rights reserved. For permission to reuse any of this content visit https://www.bmj.com/company/products-services/rights-and-licensing/permissions/

BMJ Case Report Fellows may re-use this article for personal use and teaching without any further permission.

Become a Fellow of BMJ Case Reports today and you can:

- Submit as many cases as you like

- Enjoy fast sympathetic peer review and rapid publication of accepted articles

Access all the published articles

Re-use any of the published material for personal use and teaching without further permission

Customer Service

If you have any further queries about your subscription, please contact our customer services team on +44 (0) 2071111105 or via email at support@bmj.com.

Visit casereports.bmj.com for more articles like this and to become a Fellow 